\title{
PIGMENTOS Y PINTURAS DE MINERAL DE COBRE EN LA REGIÓN DE TARAPACÁ, NORTE DE CHILE: NuEVOS DATOS PARA UNA TECNOLOGÍA PIGMENTARIA PREHISPÁNICA
}

\author{
Marcela Sepúlveda ${ }^{1}$, Valentina Figueroa ${ }^{2}$ y José Cárcamo ${ }^{3}$
}

\begin{abstract}
Resumen
La explotación de los recursos minerales de cobre en el norte de Chile implicó la producción de pigmentos azules y verdes identificados, hasta ahora, en pintura rupestre, en nódulos y en estado de polvo al interior de contenedores de cuero provenientes de distintos cementerios de la cuenca del río Loa, región de Antofagasta, desierto de Atacama. En este artículo, se presentan los primeros resultados de análisis físico-químicos de pintura verde aplicada sobre distintos soportes de cuero: máscaras, tocado y carcajes, de la región de Tarapacá, situada más al norte. Comparados a nuestros resultados previos, estos nuevos análisis nos permiten ampliar la discusión relativa a la producción e intercambio de estos pigmentos a base de minerales de cobre para períodos tardíos en el norte de Chile.
\end{abstract}

Palabras claves: pigmentos - minerales de cobre - región de Tarapacá norte de Chile.

The extraction and use of copper ore in northern Chile enabled the production of blue and green pigments identified to date in rock paintings. These pigments have also been found as nodules and in powdered form inside leather pouches that were discovered in several cemeteries in the Loa River basin in the Antofagasta region of the Atacama Desert. This article presents the initial results of physicalchemical analyses performed on selected samples of green paint found further north, in the Tarapacá region. The paint had been applied to different supports made of animal hide, including masks, headdresses and quivers. When compared to our previous results, these new analyses allow us to expand the discussion of the production of these copper-ore based pigments during late periods in northern Chile and the interactions their presence implies.

Key words: pigments - copper minerals - Tarapacá region - northern Chile.

Recibido: julio 2014. Aceptado: octubre 2014.

\section{* Introducción}

El desierto de Atacama del norte de Chile destaca por su particular concentración de minerales, los que siguen siendo explotados en la actualidad. Investigaciones desarrolladas en los últimos años han permitido demostrar la profunda antigüedad de la minería en esta área, tras los hallazgos realizados en relación con la explotación de óxidos de hierro en la mina de San Ramón 15, en la costa de la región de Antofagasta (Salazar et al. 2011a). Sin embargo, el desierto de Atacama es mayormente conocido por sus minas de cobre, las que también fueron explotadas desde tiempos prehispánicos (Núñez 1999, 2005; González y Westfall 2008; Salazar 2002, 2008; Westfall y González 2010; Salazar et al. 2010, 2013, Figueroa et al. 2013). En este contexto las tecnologías lapidaria (García-Albarido 2007; Rees 1999; Carrión 2010; Soto 2010) y metalúrgica (Figueroa et al. 2010; Salazar et al. 2011b; Cifuentes 2014) produjeron diversos bienes que formaron parte de importantes redes de intercambio (Núñez 1987 y 1999).

El análisis de pinturas rupestres y pigmentos de contextos funerarios de la región de Antofagasta en el desierto de Atacama permitió desde hace algunos años identificar el uso de distintos minerales de cobre para su producción (Sepúlveda 2006; Sepúlveda y Laval 2010; Sepúlveda et al. 2013a). Más aún, la determinación de la composición química de los pigmentos ha hecho posible dar cuenta de conocimientos específicos sobre dichas materias primas, en relación también con las producciones lapidarias y me-

\footnotetext{
1 Departamento de Antropología, Universidad de Tarapacá, Arica, CHILE. Email: marcelaasre@gmail.com/msepulveda@uta.cl

2 Instituto de Investigaciones Arqueológicas y Museo R.P. Gustavo Le Paige s.j., Universidad Católica del Norte, San Pedro de Atacama, CHILE.Email: vfigueroa@ucn.cl

3 Laboratorio de Análisis e Investigaciones Arqueométricas, Universidad de Tarapacá, Arica, CHILE. Email: jcarcamo@uta.cl
} 
talúrgicas desarrolladas en esta región desde el período Formativo hasta el período Colonial. Pese a carecer aún de elementos que permitan reconstruir el panorama integral de la fabricación, la organización espacial de la producción, la distribución y uso de los pigmentos a base de minerales de cobre, planteamos que esta tecnología fue inherente a las cadenas operativas mineras y que, por lo tanto, debe ser tratada con la misma importancia que la lapidaria y la metalurgia. Con nuestros primeros resultados propusimos la posibilidad de reconstruir el proceso de producción de pigmentos en relación a estas otras dos tecnologías (Sepúlveda et al. 2013a). Todas ellas comparten, en efecto, las primeras fases de sus cadenas operativas, es decir, el aprovisionamiento de los minerales de cobre a partir de las prospecciones mineras, la extracción mineral y hasta, probablemente, las últimas etapas de reducción del mineral. En consecuencia, la producción de pigmentos forma parte de un proceso más amplio, el que estuvo articulado con la lapidaria y/o la metalurgia, lo que demuestra que desde tiempos prehispánicos existió una alta especialización en el trabajo de los minerales de cobre. Las diferentes etapas del proceso productivo, que van desde la extracción hasta la selección, pasando por diversas fases de chancado y molienda, han sido descritas en detalle, en lo que respecta a la minería prehispánica de la vertiente occidental de la circumpuna de Atacama, en el distrito prehispánico del Abra (Salazar 2002, 2003-2004, 2008; Salinas y Salazar 2008). Por su parte, las fuentes coloniales entregan valiosas descripciones que dan cuenta de la especialización de las primeras etapas de las cadenas productivas mineras. García de Llanos (1609) describe diferentes operarios cateadores, barreteros, pirquiri, morrongo, apiri, chanqueadores, palladores, arrieros- con tareas bien específicas dentro de la mina, lo que da luces sobre el nivel de especialización de las faenas mineras en la región (García de Llanos 1983 [1609] en Figueroa et al. 2013).

Adicionalmente, la información contextual de las muestras analizadas, junto a la información arqueológica y etnohistórica, nos ha permitido proponer el desarrollo de una tecnología pigmentaria local que debió aprovechar las abundantes fuentes de minerales de la región y una tradición minera milenaria (Salazar 2002, 2008; Salazar et al. 2010, 2011a, 2013), además de participar de importantes redes de intercambio que involucraron la circulación de polvos, pigmentos y pinturas de cobre. En consecuencia, hemos planteado que un mayor estudio de la tecnología pigmentaria debía permitir discutir las formas de interacción que ocurrieron en el pasado en el desierto de Atacama (Sepúlveda et al. 2013a), pero también con las regiones vecinas.

En esta ocasión, y en la continuidad de la obtención de nuestros primeros resultados en la región de Antofagasta, presentamos nuevos análisis realizados sobre muestras de pintura verde aplicada sobre distintos soportes de cuero provenientes de sitios ubicados en la costa de la región de Tarapacá situado a unos 300 km más al norte. Comparados, estos nuevos resultados nos permiten aportar elementos para indicar la existencia también en esta región de una tecnología del color basada en minerales de cobre. Pese a la identificación de distintos minerales en las pinturas, se aprecian procesos de producción semejantes que hablan de una tecnología compartida, lo que permite reafirmar la raigambre local, en el desierto de Atacama, de una práctica desarrollada con mayor fuerza sobre todo a partir del Intermedio Tardío (900-1450 DC) y sostenida hasta el siglo XVII. No obstante, algunas evidencias de épocas previas nos llevan a discutir una antigüedad aun mayor para este tipo de práctica.

En este trabajo se resumen los principales estudios sobre pigmentos y pinturas realizados en el Norte Grande en los últimos años. Posteriormente, describimos las muestras analizadas, las técnicas de análisis y nuestros principales resultados. Los datos obtenidos sobre estas pinturas verdes en base a mineral de cobre de la región de Tarapacá son integrados a los antecedentes disponibles para la región, de modo de discutir su inserción en las redes de interacción conocidas para tiempos tardíos, en todo el desierto de Atacama y regiones vecinas.

\section{* Estudios sobre pigmentos y pinturas EN EL Norte Grande}

En el norte de Chile, los análisis físico-químicos de pigmentos y pinturas rupestres son aun relativamente escasos. No obstante, en los últimos años diversos trabajos que han abordado la tecnología del color y el estudio de la minería, han considerado el análisis de pigmentos presente sobre soportes distintos a la cerámica. Entre otros se han analizado recubrimientos corporales de momias Chinchorro (Arriaza et al. 2006, 2008; Silva-Pinto et al. 


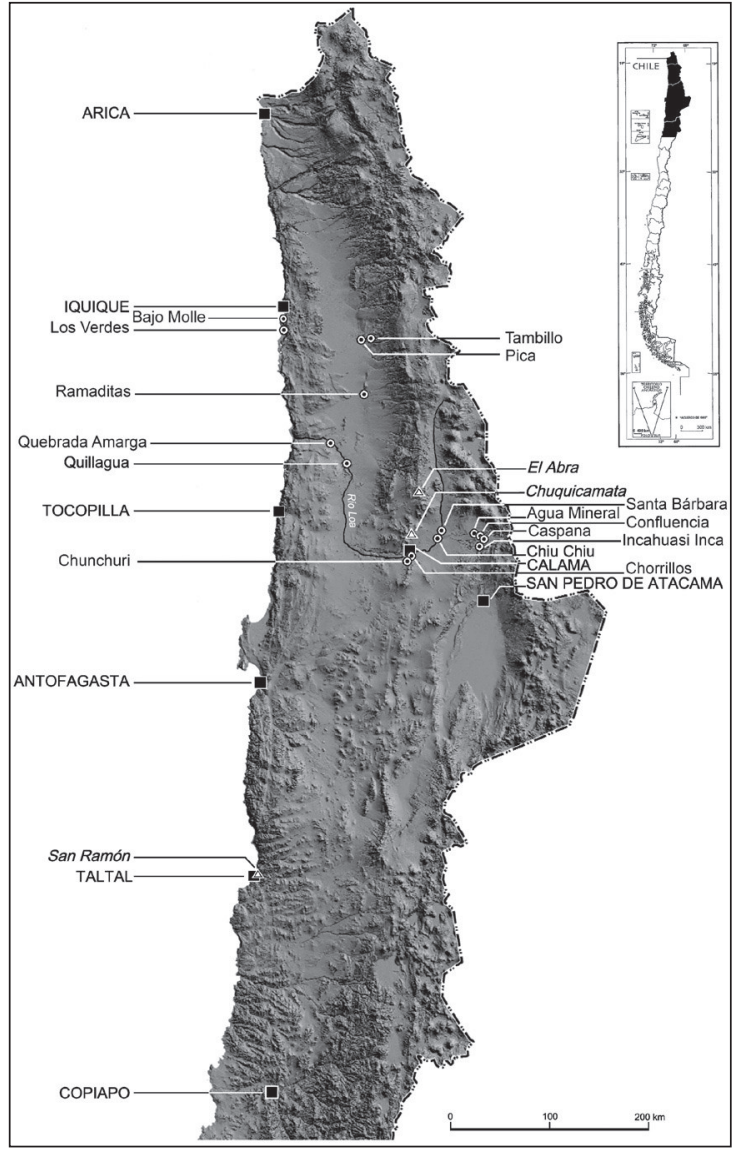

Figura 1. Mapa con los principales sitios que presentan pigmentos minerales a base de minerales de cobre.

2013; Sepúlveda et al. 2014), bloques de colores del sitio Playa Miller 7 (Sepúlveda et al. 2013b), y materias colorantes ferruginosas procedentes de sitios mineros (San Ramón 15) y habitacionales del período Arcaico Temprano, Medio y Tardío de la costa de Taltal (Salazar et al. 2011a; Coquinot y Salomon 2013, 2014). En relación con pinturas rupestres han sido realizados estudios, de norte a sur, en la precordillera de Arica en el extremo norte de Chile (Sepúlveda et al. 2012) y la cuenca del río Loa (Helwig y Wainwright 1999; Sepúlveda et al. 2010a y 2010b; Gallardo et al. 2012). En general, el aumento de trabajos sobre estos materiales, en su mayoría conciernen análisis de pinturas rojas y, en menor medida negras, siendo los trabajos sobre pigmentos y pinturas verdes y azules de la región de Antofagasta, los primeros llevados a cabo en los Andes sobre estos colores para tiempos prehispánicos (Sepúlveda y Laval 2010a; Sepúlveda et al. 2013a).
En estos recientes trabajos informamos sobre análisis elemental y mineralógico de distintas muestras de pinturas procedentes de los sitios Incahuasi Inca, Santa Bárbara, Confluencia y Quebrada Amarga, así como de pigmento en estado de polvo almacenado en saquitos de cuero de los cementerios de Chunchuri (o Dupont 1) en Calama, y de Los Abuelos en Caspana, todos en distintas áreas de la cuenca del río Loa en la región de Antofagasta (Figura 1 y 2). En dicho estudio, se combinaron la observación microscópica y la Microscopia de Electrónica de Barrido (SEM), para luego realizar análisis elementales mediante el Espectrómetro por Dispersión de Rayos X (EDX) acoplado al SEM. Finalmente, con RAMAN realizamos una caracterización molecular de los distintos minerales empleados. Es así como inicialmente identificamos el uso de óxidos de cobre, sulfatos de cobre y cloruros de cobre (Sepúlveda et al. 2010, 2013a), los que fueron posteriormente precisados como atacamita $\left(\mathrm{Cu}_{2} \mathrm{Cl}(\mathrm{OH})_{3}\right)$, clinoatacamita $\left(\mathrm{Cu}_{2} \mathrm{Cl}(\mathrm{OH})_{3}\right)$, lancita ${ }^{2}\left(\mathrm{Cu}_{4}\left(\mathrm{SO}_{4}^{3}\right)\right.$ $\left.(\mathrm{OH})_{6} \cdot 2 \mathrm{H}_{2} \mathrm{O}\right)$, bandilita $\left(\mathrm{CuB}_{2} \mathrm{O}_{4} \mathrm{CuCl}_{2} \cdot 4 \mathrm{H}_{2} \mathrm{O}\right)$, crisocola $\left((\mathrm{CuAl})_{2} \mathrm{H}_{2} \mathrm{Si}_{2} \mathrm{O}_{5}(\mathrm{OH})_{4} \cdot \mathrm{nH}_{2} \mathrm{O}\right)$ y malaquita $\left(\mathrm{Cu}_{2} \mathrm{CO}_{3}(\mathrm{OH})_{2}\right)^{2}($ Sepúlveda et al. $2013 \mathrm{a}$ ) (Tabla 1).

De acuerdo con estos resultados identificamos la producción de distintas mezclas elaboradas en base a minerales de cobre provenientes seguramente de la región, donde se encuentran de forma abundante. Los cloruros son frecuentes, por lo que resulta complejo distinguirlos. Los sulfatos también pueden provenir de fuentes minerales de la región, aunque a su vez pueden ser el producto de la alteración de las pinturas sobre las paredes, sobre todo en presencia de yeso, comúnmente identificada en la superficie conformando una pátina translúcida. Lo interesante fue observar la heterogeneidad de las fuentes, tal como lo demostraron los resultados del análisis de mineral proveniente de la mina de Incahuasi (Sepúlveda et al. 2013a).

En términos cronológicos, las muestras analizadas correspondieron a pinturas atribuidas estilísticamente al período Intermedio Tardío (Confluencia, Quebrada Amarga y Santa Bárbara) o al Tardío (Incahuasi Inca; Adán y Uribe 1995; Sepúlveda et al. 2013a). Las muestras de pigmento de Chunchuri y del cementerio Los Abuelos de Caspana también fueron atribuidas a este lapso temporal (Uhle 1912-1913 y Núñez 1966; Ayala et al. 1999, respectivamente) confirmando que la mayor cantidad 


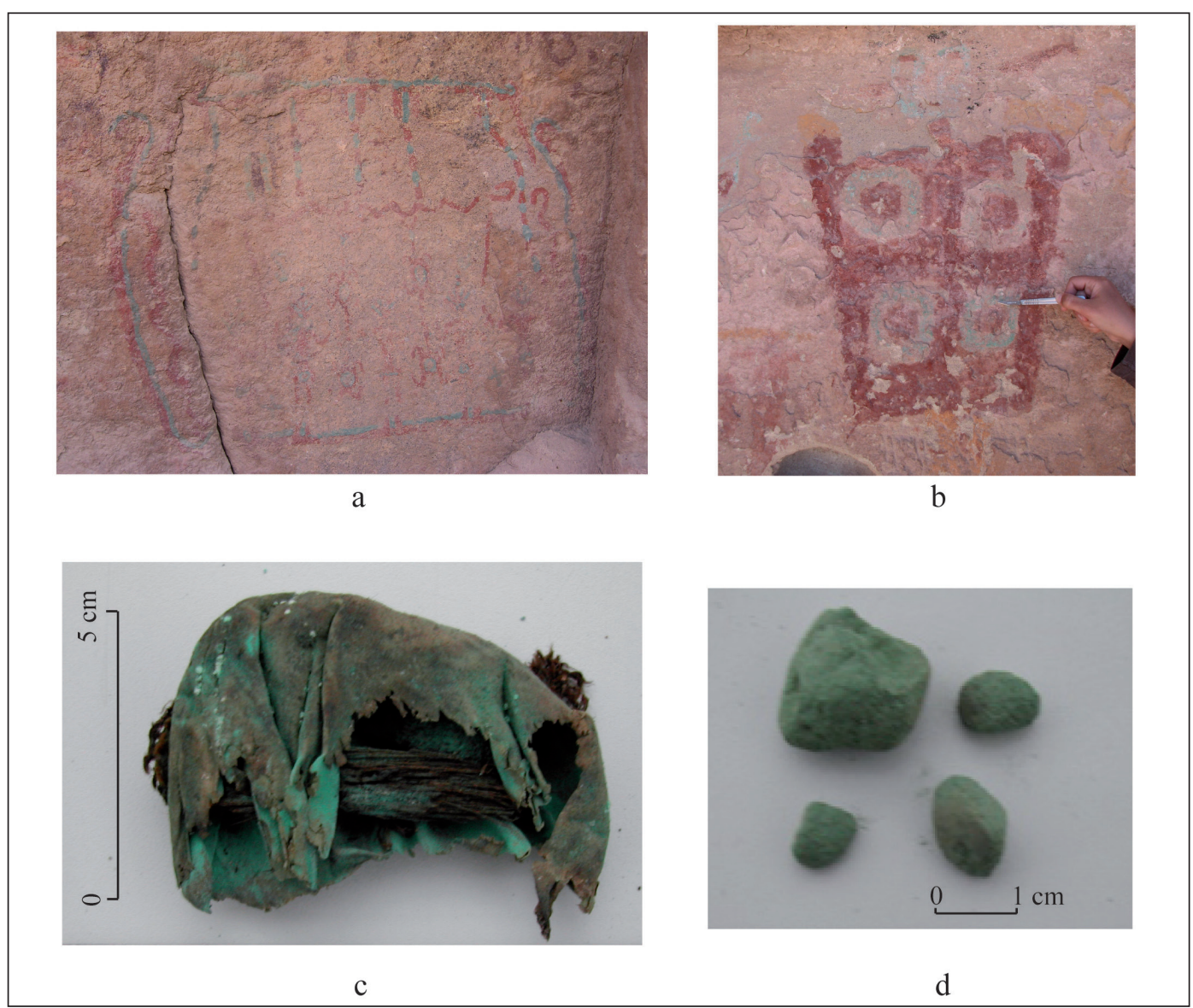

Figura 2. Pigmentos minerales de cobre en diferentes soportes: a) Incahuasi Inca; b) Santa Bárbara; c) Caspana; d) Chunchuri.

de muestras es atribuida a los períodos tardíos de la secuencia regional. Con estos antecedentes propusimos la existencia de una tecnología referida a la producción de pigmentos como algo propio de la cuenca alta del río Loa, en tiempos tardíos, coincidente con la presencia de importantes áreas de aprovisionamiento de mineral de cobre (Sepúlveda et al. 2013a).

\section{* Descripción de las muestras, técnicas de ANÁLISIS Y RESULTADOS}

\section{Corpus de muestras arqueológicas}

Para el presente trabajo, se analizaron en total cinco muestras: dos máscaras de cuero del sitio Los Verdes (Sanhueza 1985), dos carcajes del sitio Bajo Molle y un tocado de cuero perteneciente al llamado "Señor de Pica" o Señor de Bajo Molle (Moragas 1995), contexto que a su vez fue encontrado en Bajo Molle y que forma parte de la colección Anker Nielsen depositada en el Museo Regional de Iquique (Tabla 2). Hasta ahora, a partir de nuestra revisión de colecciones de la región, estos soportes son los únicos en presentar evidencias claras de pintura verde. Los Verdes corresponde a un sitio funerario costero atribuido al Intermedio Tardío o Desarrollos Regionales (Sanhueza 1985), con evidencias de una estructura rectangular, sin embargo, asociada a la tercera época de ocupación de la costa, interpretada como la consolidación de una tradición costera iniciada o contemporánea del período Formativo y sostenida hasta tiempos coloniales y republicanos (Urbina et al. 2011: 91). El sector de Bajo Molle -igualmente costero- presenta distintos asentamientos cuyas ocupaciones van desde el período Arcaico Chinchorro hasta el Intermedio Tardío 


\begin{tabular}{|l|l|l|l|l|l|}
\hline Muestra & Localidad & Color & Forma física & SEM-EDX & Raman \\
\hline Santa Bárbara 1-9 & Alto Loa & Verde pálido & Pintura & $\mathrm{Cu}, \mathrm{Cl}$ & Atacamita, poca eriocalcita y yeso \\
\hline Incahuasi o1 & Río Salado & Verde & Pintura & $\begin{array}{l}\mathrm{Cu}, \mathrm{Cl}, \mathrm{S}, \mathrm{Al}, \mathrm{Si}, \\
\mathrm{Mg}, \mathrm{Na}, \mathrm{Ca}\end{array}$ & Clinoatacamita y yeso \\
\hline Incahuasi 03 & Río Salado & Azul & Pintura & $\mathrm{Cu}, \mathrm{Cl}$ & Atacamita y yeso \\
\hline Incahuasi 05 & Río Salado & Azul & Pintura & & Clinoatacamite \\
\hline Quebrada Amarga o1 & Loa Inferior & Verde pálido & Pintura & & Bandylita y yeso \\
\hline Confluencia o1 & Río Salado & Verde & Pintura & $\begin{array}{l}\mathrm{Cu}, \mathrm{Cl}, \mathrm{S}, \mathrm{Al}, \mathrm{Si}, \\
\mathrm{Na}, \mathrm{K}\end{array}$ & Bandylita \\
\hline Confluencia o9 & Río Salado & Verde & Pintura & $\mathrm{Cu}, \mathrm{Cl}, \mathrm{Al}, \mathrm{Mg}, \mathrm{Fe}$ & Bandylita \\
\hline Dupont 1 & Loa Medio & Verde pálido & Polvo & $\mathrm{Cu}, \mathrm{S}$ & Langita \\
\hline Caspana 1 & Río Salado & Verde & Polvo & $\mathrm{Cu}, \mathrm{Cl}$ & Atacamita \\
\hline Incahuasi fuente & Río Salado & Verde y azul heterogéneo & Fragmentos roca & $\mathrm{Cu}, \mathrm{S}$ & Crisocola y Malaquita \\
\hline
\end{tabular}

Tabla 1. Sitios arqueológicos de la región de Antofagasta analizados que presentan pigmentos minerales a base de mineral de cobre.

\begin{tabular}{|l|l|l|l|l|l|l|}
\hline Muestra & Localidad & Sitio & Color & Forma física & SEM-EDX & Raman \\
\hline LV1 & Costa Tarapacá & Los Verdes & Verde & Pintura & Si, S, Ca, Al, Mg, Cu & Antlerita \\
\hline LV 2 & Costa Tarapacá & Los Verdes & Verde & Pintura & P, S, Si, Ca, Cu, $\mathrm{Al}, \mathrm{Mg}, \mathrm{Fe}$ & Antlerita \\
\hline Casco 1 & Costa Tarapacá & Bajo Molle & Verde pálido & Pintura & - & Brocantita \\
\hline Carcaj 2 & Costa Tarapacá & Bajo Molle & Verde & Pintura & - & Atacamita \\
\hline Carcaj 3 & Costa Tarapacá & Bajo Molle & Verde & Pintura & Cu, Al, Si & Atacamita \\
\hline
\end{tabular}

Tabla 2. Sitios arqueológicos de la región de Tarapacá analizados que presentan pigmentos minerales a base de mineral de cobre.

(Moragas 1995), no obstante, los objetos aquí analizados son atribuidos a este último período.

Las máscaras de cuero, probablemente de lobo marino, y de forma trapezoidal, son polícromas pues presentan colores verde, blanco, negro, rojo y amarillo. En la primera máscara (LV.1) estos colores forman motivos rectangulares al interior de los cuales se disponen dos cruces negras con bordes escalerados, pintadas negras en su interior y verde en su exterior, ambos colores separados por un trazo blanco (Figura 3). Estas cruces finalizan en su parte superior e inferior con unos ganchos de color negro rodeados por una línea blanca. Las cruces son segmentadas en cuatro partes por líneas blancas. A cada lado de los motivos centrales en cruces se encuentra un rectángulo bícromo con una línea diagonal escalerada separando dos porciones pintadas en un caso en rojo, en otro caso en amarillo. Sobre las cruces y los rectángulos amarillos y rojos se encuentra una franja horizontal de triángulos negros. Sobre ella y en sentido inverso se encuentra una franja de triángulos blancos. La segunda máscara (LV.2) presenta los mismos motivos en cruz al centro aunque sin los ganchos. Se aprecian también los mismos rectángulos bícromos amarillo y rojo a cada lado, sin las franjas de triángulos superiores. Ambas máscaras presentan flecos cortados en el cuero en la parte superior (Figura 3). Si bien, la atribución cronológica del sitio es muy amplia, desde finales de los Desarrollos Regionales, o período Intermedio Tardío, hasta el período incaico (Sanhueza 1985), como hipótesis podemos situar este contexto a comienzos del período Intermedio Tardío. Futuros fechados confirmarán la adscripción cronológica.

El casco del "Señor de Pica" corresponde a un fragmento de cuero tubular para rodear la cabeza con una apertura mayor a nivel del rostro ${ }^{4}$ (Figura 4). El material del casco

4 Lautaro Núñez fue quien realizara el primer montaje del Museo Regional de Iquique. En esa oportunidad él señaló dicho contexto como un "Señor de Pica", por las similitudes con los contextos excavados en el cementerio Pica-8. Sin embargo, el contexto no es de "Pica", sino de Bajo Molle (comunicación personal Núñez 2014). 


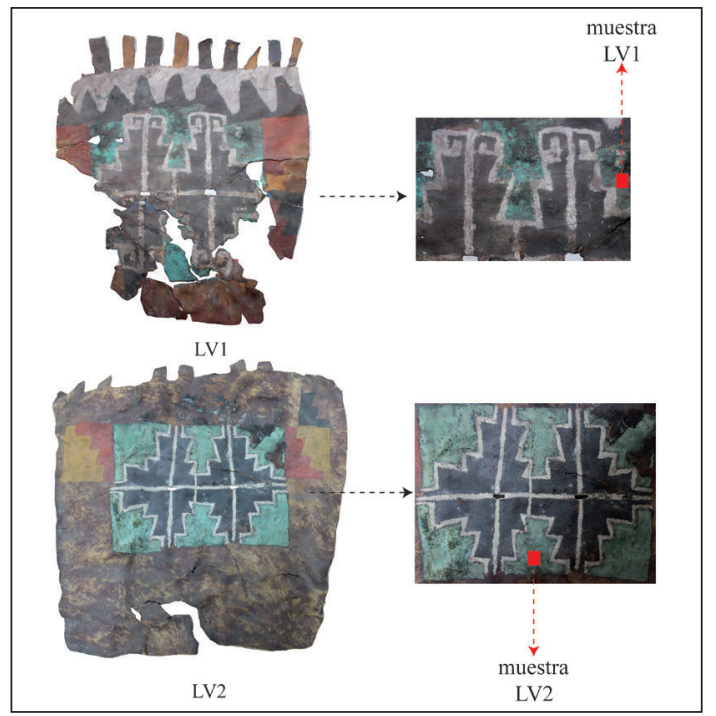

Figura 3. Máscaras fabricadas en cuero de lobo marino policromas procedentes de Los Verdes (Iquique, Región de Tarapacá), conservadas en el Museo Regional de Iquique.

fue fabricado probablemente con cuero de lobo marino. En la parte superior posee otro fragmento de cuero horizontal cocido al primer gran fragmento. A nivel de la costura se encuentran amarrados a un entramado de fibra vegetal, vellones teñidos con pigmento rojo, semejantes a los identificados en los gorros atacameños (comunicación personal Agüero 2014). En la parte trasera del casco, distintas plumas de parina se encuentran atadas sobresaliendo de la parte horizontal superior del casco. Varias de éstas se encuentran en mal estado. Dos conjuntos de hilados torcidos, uno azul, otro blanco, rodean el casco debajo de los vellones, para sostener las plumas. El casco presenta motivos geométricos en bandas pintados de verde. La decoración del casco en color verde se localiza en la parte tubular que rodea el rostro, es decir hacia la sección delantera del casco (Figura 4).

Los dos carcajes de cuero, fabricados a partir de la piel de un zorro, provienen del sitio de Bajo Molle. ${ }^{5}$ Ambos presentan en la parte superior, hacia la apertura por donde se introducen los dardos, una franja pintada de color verde. En un caso la pintura fue aplicada directamente sobre el

5 Presentan la referencia "Bm 1279 y Bm1296" que representa a las iniciales de Bajo Molle junto a un número de inventario. Dichas referencias son propias al inventario de la colección Anker Nielsen.

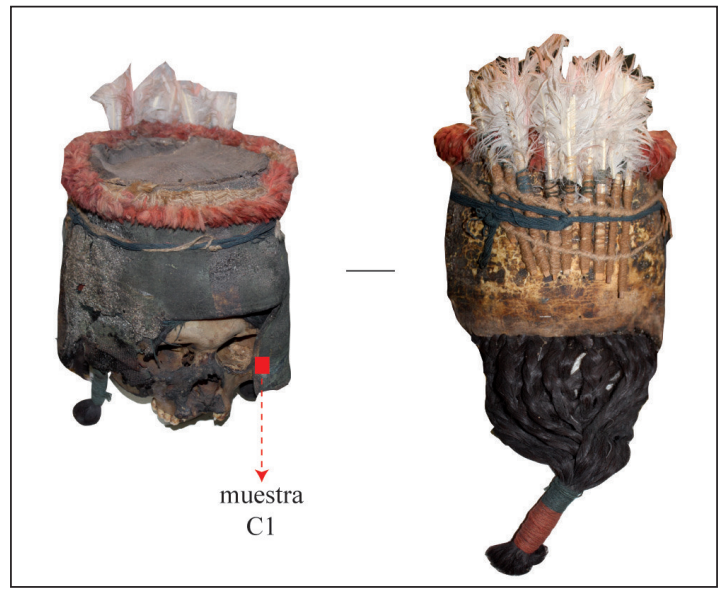

Figura 4. Casco fabricado en cuero procedente del sitio costero de Bajo Molle (Iquique, Región de Tarapacá), conservado en el Museo Regional de Iquique.

cuero, en el otro caso sobre pelos, siendo entonces fácilmente desprendible. Si bien los tres objetos provienen del mismo sitio y presentan características similares en cuanto a la aplicación del color verde, no podemos por el momento señalar con seguridad que éstos provengan del mismo contexto (Figura 5).

\section{* Protocolo analítico}

Las muestras fueron observadas por lupa binocular a distintos aumentos. Luego fueron observadas y analizadas también mediante Microscopio Electrónico de Barrido acoplado a un Sistema de Dispersión de Rayos X (SEMEDX), marca JEOL- Neoscope II (Figura 6). Finalmente, las muestras fueron analizadas mediante espectrometría Raman. Estos espectros Raman fueron registrados en un equipo Raman Renishaw InVia Reflex, con fuentes de excitación de 532, 633 and $785 \mathrm{~nm}$, equipado con un microscopio Leica y un cámara CCD enfriada eléctricamente. Las medidas micro-Raman fueron calibradas usando una placa de silicio y un objetivo de 50X. De 1 a 20 barridos con tiempos entre 10 y 30 s fueron utilizados para obtener los espectros. El poder del láser 785 fue ajustado entre 0,001 y $1 \%$. Los espectros fueron registrados en la región comprendida entre 200 y $1800 \mathrm{~cm}^{-1}$. Los espectros reproducibles de las muestras de iglesias fueron escaneadas directamente sobre las muestras depositadas 
sobre una placa de cuarzo, atenuando o apagando de esta manera la fluorescencia natural de las muestras. Los programas Wire 3.4 y GRAMS/AI 8 fueron utilizados para analizar la información espectral. Los espectros obtenidos fueron comparados con la base de datos y RRUFF (Downs 2006).

\section{$*$ Resultados}

Los resultados del análisis de las pinturas verdes $\mathrm{LV}_{1}$, $\mathrm{LV}_{2}, \mathrm{C}_{1}, \mathrm{C}_{2}$ y $\mathrm{C}_{3}$ indican que todas presentan una composición heterogénea conformada por granos verdes opacos y/o brillantes de distintos tamaños, negros y translúcidos de gran tamaño (200 ctm). ${ }^{6} \mathrm{Al}$ no poder metalizar e intervenir las muestras en esta instancia, la observación por Microscopio de Barrido fue bastante limitada, no obstante pudimos identificar ciertos elementos. En los casos que obtuvimos resultados, esto se logró porque la capa de pintura aplicada resultaba ser bastante gruesa como para cubrir el soporte de cuero. Así pudimos observar la presencia de diferentes compuestos de cobre en las muestras LV1, LV2 y Carcaj 3, tales como óxidos de cobre y sulfatos de cobre. También identificamos la presencia, en ciertos casos, de aluminosilicatos que los engloban o comprenden, pudiendo corresponder a algún tipo de carga y/o aglutinante (Figura 7.1 y 7.2). También en el caso de LV2 observamos la presencia de fosfato y calcio, posiblemente asociados al cuero.

Por Raman, los resultados obtenidos indican, la identificación de distintos minerales, tales como brocantita $\left(\mathrm{Cu}_{4} \mathrm{SO}_{4}(\mathrm{OH})_{6}\right)$, antlerita $\left(\left(\mathrm{Cu}^{2+}\right)_{3} \mathrm{SO}_{4}(\mathrm{OH})_{4}\right)$ y atacamita $\left({ }^{4} \mathrm{Cu}_{2} \mathrm{Cl}(\mathrm{OH})_{3}\right)$ (Tabla 2; Figura 7.3$)^{4}$. De esta manera, pudimos reconocer en los casos analizados la presencia de mezclas a base de minerales de cobre combinados con otros compuestos, aún imprecisos, para producir una pintura aplicable sobre cueros. Sin la presencia de un aglutinante orgánico o mineral, de tipo arcilla, un polvo a base de mineral de cobre no es suficiente para lograr una pintura con consistencia y capaz de adherirse al cuero.

6 El tamaño es relativo. La granulometría grande se refiere al gran tamaño de los granos que componen las mezclas en relación a otras muestras analizadas donde los diferentes elementos miden menos de 10 ctm.

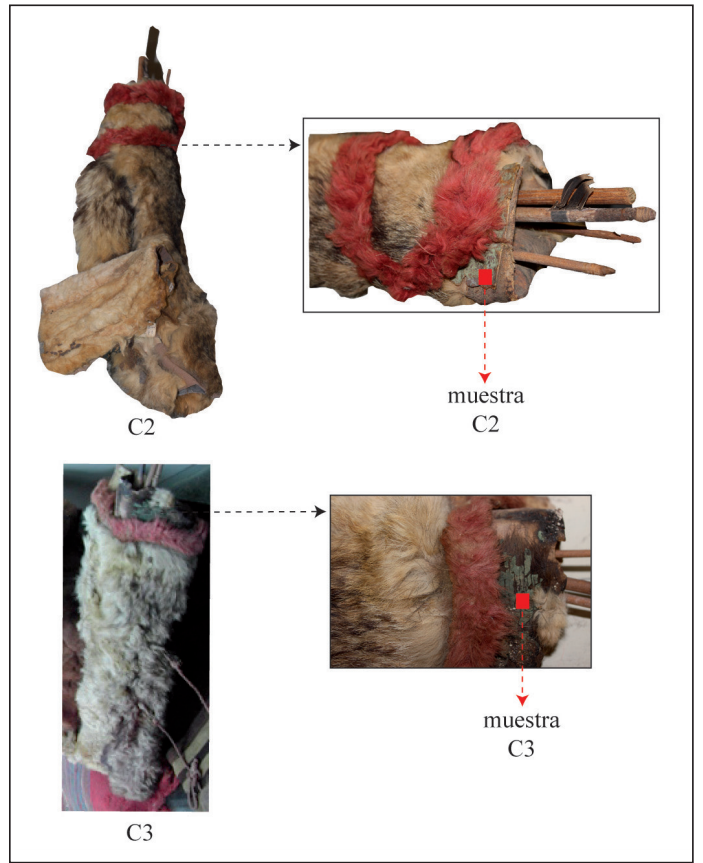

Figura 5. Dos carcajes del sitio costero de Bajo Molle (Iquique, Región de Tarapacá), conservados en el Museo Regional de Iquique.

\section{Pigmentos y pintura en base a mineral de cobre: tecnología e interacción en tiempos tardíos}

A partir del análisis elemental y mineral realizado sobre distintas muestras de pintura verde pudimos previamente identificar el uso de minerales de cobre para la producción de pinturas en el desierto de Atacama (Sepúlveda y Laval 2010a, Sepúlveda et al. 2013a), hecho que hoy ampliamos a la región de Tarapacá. Consecuentemente, estos resultados permiten definitivamente ampliar la utilidad y uso de los minerales de cobre explotados en el norte de Chile para una función poco enunciada, hasta ahora, y particularmente inédita para el área Centro Sur Andina. La pintura puede ser encontrada en su calidad de mezcla aglutinada como es el caso de aquella asociada al entierro del cementerio de Chunchuri, de polvo, como es el caso de Caspana, puede ser encontrada como pintura rupestre (Quebrada Amarga, Incahuasi, Santa Bárbara y Confluencia) o bien también en diversos soportes tales como los cueros aquí analizados (Los Verdes, Bajo Molle).

En términos moleculares, resulta interesante la identificación en la región de Tarapacá de minerales de cobre distin- 


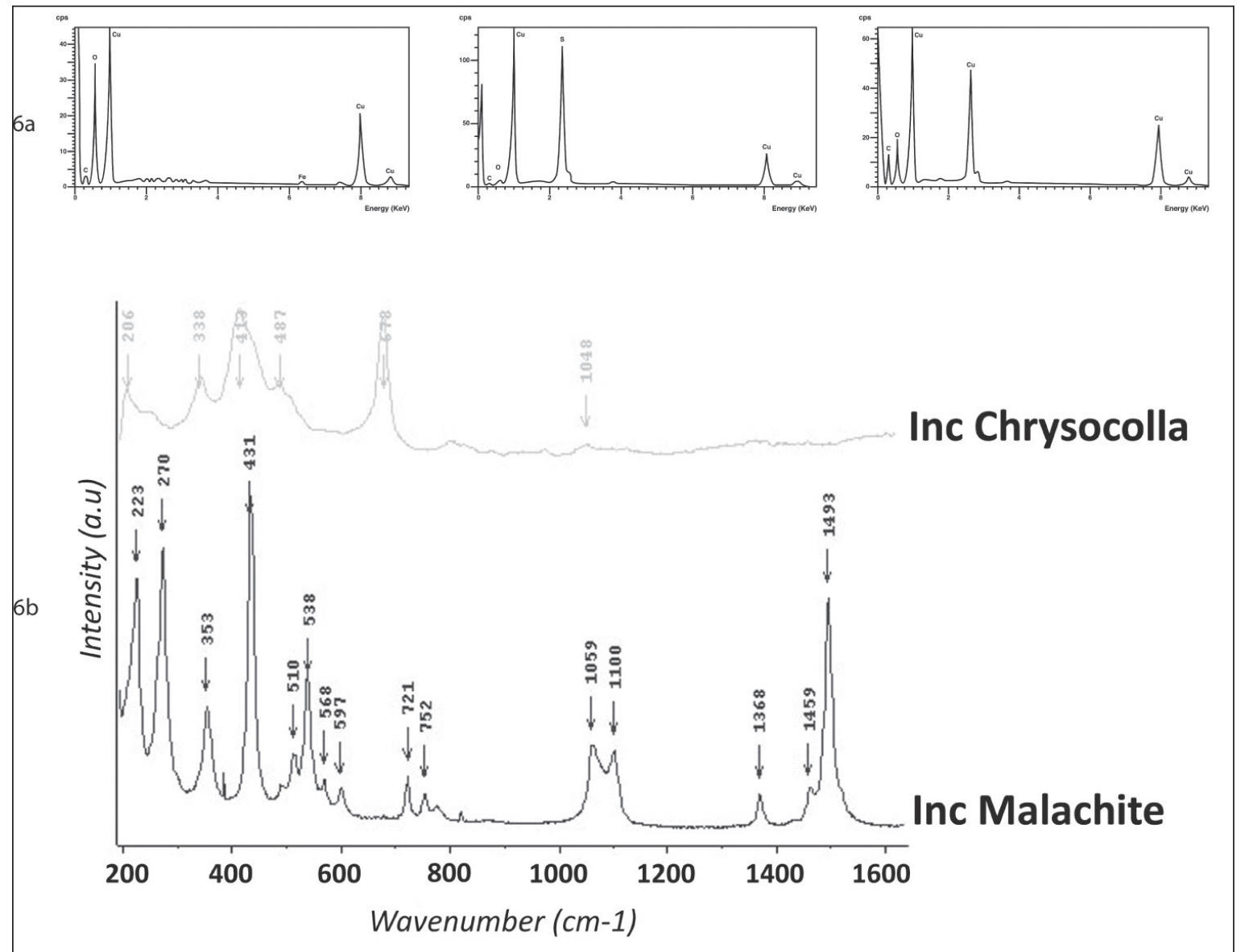

Figura 6. Protocolo analítico del estudio de pigmentos a base de minerales de cobre: a. Ejemplos análisis SEM-EDX de muestra INCo3; b. Espectro Raman de fragmentos de mina de Incahuasi.

tos a los identificados previamente en la de Antofagasta. Salvo en el caso de los dos carcajes pintados con una pintura a base de atacamita, los otros soportes de cuero de Los Verdes y El Señor de Pica presentan una pintura a base de antlerita o brocantita. Estos últimos casos podrían ser originalmente del mismo mineral de antlerita, al ser metaestable a temperatura ambiente y al poder alterarse a altas temperaturas en brocantita. No obstante, pese a estos resultados por el momento no es posible, con los elementos disponibles, distinguir o asimilar producciones tecnológicas entre estas dos regiones sólo en base a la identificación de minerales de cobre distintos. Se suma a esto el hecho de que las minas de cobre del desierto de Atacama suelen presentar una importante diversidad de minerales de acuerdo a los procesos supérgenos característicos de esta zona (Neumann 1973; Chávez 2000). En consecuencia, resulta imposible por ahora atribuir la extracción de minerales de cobre para la producción de pigmento a una fuente específica. Además, mientras no hayamos finalizado el registro sistemático de colecciones y pinturas rupestres de todo el norte de Chile (regiones de Arica y Parinacota, Tarapacá, Antofagasta hasta Atacama) resultará difícil cuantificar precisamente el uso de pigmento y pintura verde y así evaluar su mayor significancia. Hasta ahora, la mayor concentración de pinturas se localiza en la cuenca del río Loa, en la región de Antofagasta. En Tarapacá, salvo el caso de Tambillo, los demás soportes refieren a artefactos móviles que bien pudieron ser fabricados o pintados en otras regiones previo su entierro en la costa de Iquique. Así, de momento carecemos de argumentos para plantear en esta región una tecnología propia y distinta a la identificada previamente en la cuenca del río Loa. 
Pigmentos y pinturas de mineral de cobre en la región de TARApacá, norte de Chile: NUEVOS DATOS PARA UNA TECNOLOGÍA PIGMENTARIA PREHISPÁNICA

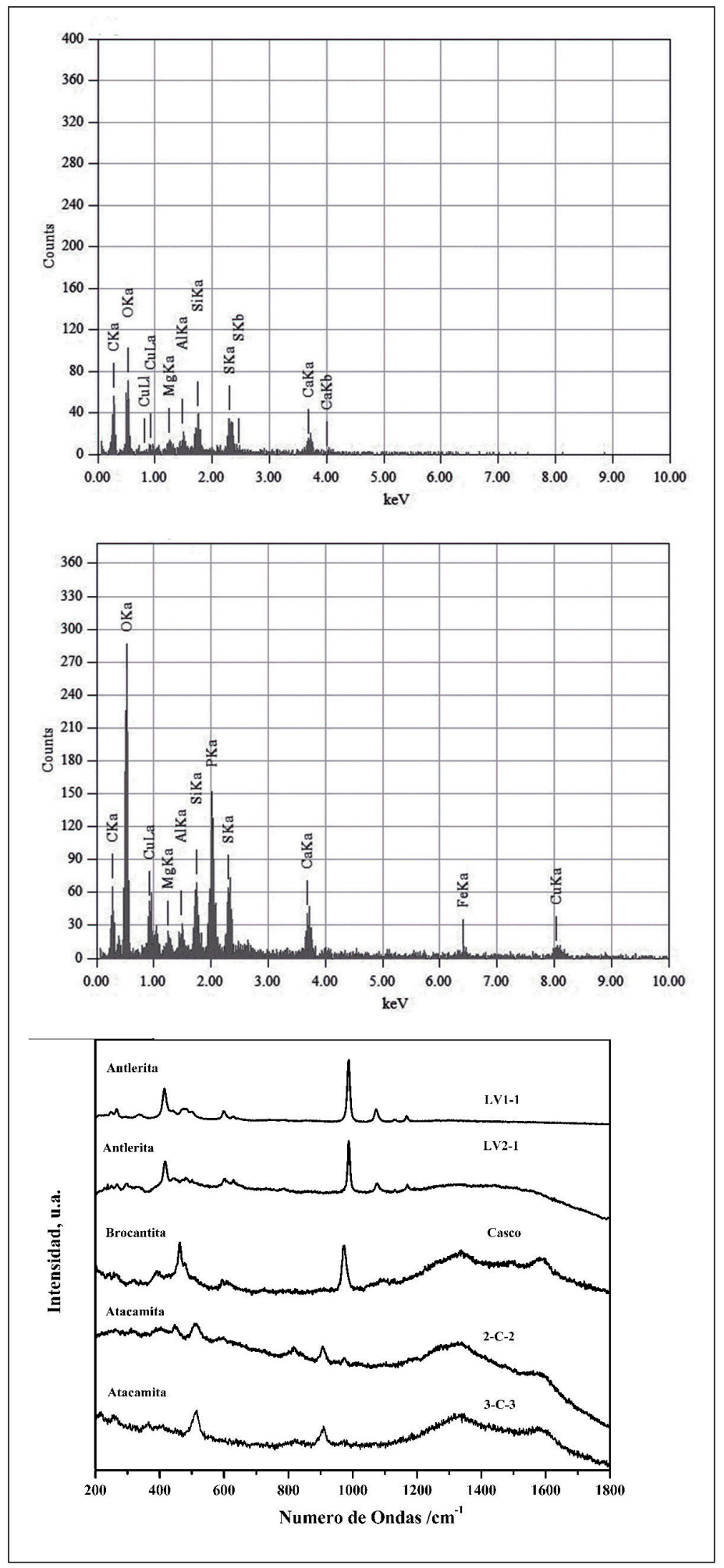

Figura 7. Resultados de los análisis SEM-EDX y Raman de las muestras de la Región de Tarapacá. 7.1. Espectro SEM LV1; 7.2. Espectro SEM LV2; 7.3. Espectros Raman de todas las muestras. 
Al problema del origen de la tecnología pigmentaria de los minerales de cobre, se suma que resulta difícil precisar su antigüedad. Numerosas minas, desmontes y artefactos asociados a las operaciones mineras son las evidencias de un contexto regional de antigua tradición minera en el norte de Chile (Salazar 2002, 2008; Figueroa et al. 2013). Precisamente, en ese contexto se inserta el hallazgo del minero, el Hombre de Cobre, en el sector de Chuquicamata, situado en proximidad a la cuenca del río Loa, junto a diferentes artefactos propios del equipamiento de un minero como martillos enmangados con cuero y madera, un capacho y un canasto de cestería (Bird 1977-78 y 1979; Craddock 1990). Esta y otras momias, como la encontrada por la misión francesa dirigida por Créqui-Monfort, indican que la tecnología minera se encuentra perfectamente desarrollada por los menos desde el período Formativo (Figueroa et al. 2013). No obstante, esta tecnología podría remontarse inclusive a tiempos arcaicos en contextos Chinchorro. En efecto, recientemente hemos identificado la presencia de mineral de cobre al interior de la momia de una niña del sitio Estación Sanitaria, en la ciudad de Arica (Sepúlveda et al. 2014). El color verde se aprecia también sobre el rostro de figurinas de arcilla con el rostro modelado pintado del sitio de Patillo, cerca de la ciudad de Iquique (Moragas 1995). Estos hallazgos hacen, entonces, posible pensar en retrotraer el manejo de conocimiento específicos sobre el uso de minerales de cobre y su empleo para la preparación de pintura, al período Arcaico.

Si bien no es posible resolver la antigüedad de la tecnología del color en base a los minerales de cobre del norte de Chile, sumamos con este trabajo más antecedentes para discutir su importancia al menos en tiempos tardíos. En relación a los objetos aquí presentados resulta relevante señalar que los contextos de origen de los objetos analizados procedentes de la región de Tarapacá pertenecen todos al período Intermedio Tardío. El sitio Los Verdes, de acuerdo al patrón arquitectónico identificado es atribuido a dicho período (Urbina et al. 2011), si bien no es posible desconocer la presencia en el sector de cerámica Formativa (Uribe 2009). La iconografía de las máscaras es atribuida al Intermedio Tardío, de igual forma que el casco del "Señor de Pica". Es de primera importancia mencionar entonces la existencia de una tradición minera prehispá-

\footnotetext{
7 Conservado actualmente en el American Museum of Natural
} History de Nueva York. nica tarapaqueña durante este período. Para el Intermedio Tardío se reconoce actualmente una instalación minerometalúrgica ubicada en Collahuasi (Berenguer 2007; Berenguer y Cáceres 2008; Berenguer et al. 2011; Urbina 2012) y en la Quebrada de Tarapacá (Zori y Tropper 2010; Zori 2011). Tanto las fechas obtenidas del poblado Collahuasi 37, como la presencia de cerámica preincaica en el sitio, ratifican que aquí en efecto funcionó una instalación durante el Intermedio Tardío, muy posiblemente vinculada con la explotación y procesamiento de minerales de cobre, la cual fue posteriormente incorporada y transformada por el Estado incaico para insertarla dentro de su propia economía política (Lynch y Núñez 1994; Berenguer et al. 2011; Salazar et al. 2013; Urbina 2012). Si bien queda pendiente ahondar acerca de las cadenas operativas cupríferas del Intermedio Tardío e Inca del Distrito Collahuasi, y a su vez, de las instalaciones previas a la ocupación incacolonial de Tarapacá Viejo y de los otros sitios metalúrgicos descritos para la Quebrada de Tarapacá, la presencia de una producción de cobre tarapaqueña es manifiesta (Mille et al. 2013). En esa perspectiva, será de primera importancia realizar estudios más detallados que permitan correlacionar la utilización de los minerales de cobre procedentes de las menas tarapaqueñas en los contextos prehispánicos de la región de Tarapacá.

La utilización de minerales de cobre destinados a la preparación de pigmentos formó parte de un entramado común con otras tecnologías, como la lapidaria y la metalurgia, muy representativas del norte de Chile. Estas últimas se insertaron fuertemente en el tráfico caravanero regional a través del excedente minero para recibir a cambio productos del noroeste argentino y del altiplano boliviano (Núñez 1987; Núñez y Dillehay 1995; Salazar 2002). Este proceso es muy evidente desde el Intermedio Tardío, si bien no podemos descartar su importancia en períodos previos. Testimonios de este tráfico son, por ejemplo, algunas pinturas que hemos atribuido al período Intermedio Tardío, como Agua Mineral (Sepúlveda 2006) o Quebrada Amarga (Sepúlveda et al. 2013a), así como las bolsas de cuero con pigmento verde en polvo provenientes de los cementerios de Chunchuri (Calama) y Los Abuelos de Caspana. Posteriormente, en tiempos tardíos, se reconoce el uso de pintura en chullpas de la zona de Carangas (Gisbert 1994) y en el sitio de Tambillo, donde se observa una figura antropomorfa con una túnica ajedrezada pintada de verde y rojo, cuyo contexto 
se vincularía con la explotación incaica de Collahuasi, siendo cercano a un ramal del camino inca (Salazar et al. 2013). Más al sur, ejemplos de pintura verde se encuentran en el sector conocido como Finca Chañaral también relacionado con el camino incaico que cruza la región de Copiapó (Cervellino y Sills 2001; González 2007).

En consecuencia, si se combinan estos datos con la información proveniente de las otras tecnologías lapidaria y metalúrgica, es lógico pensar que la referida a la producción de pigmentos participó igualmente de redes de intercambio, a partir del Intermedio Tardío al menos, articulando distintas áreas del desierto de Atacama con regiones vecinas. Este tráfico habría perdurado en tiempos coloniales, tal como lo mencionan referencias etnohistóricas (Siracusano 2005; Sepúlveda et al. 2013a) que indican que los materiales provenientes de las minas de cobre de la cuenca del río Loa eran particularmente apreciados, alimentando importantes rutas del tráfico de minerales y pigmentos durante los siglos XVI a XVIII, al menos. La Colonia no habría hecho más que aprovechar el tráfico pre-existente mediante su rearticulación en torno a nuevas rutas. La presencia de pintura verde a base de minerales de cobre -en particular atacamita - en la Virgen de Copacabana del siglo XVI, indica que su uso seguía vigente en esa época (Tomasini et al. 2012). Más aún, la presencia de antlerita en las iglesias coloniales en la precordillera de Arica en el extremo norte de Chile, confirma su permanencia durante el siglo XVIII, habiendo sido producto de intercambio posiblemente a través de la ruta Potosí-Arica (Guzmán et al. 2014).

\section{* Comentarios finales}

La caracterización físico-química de los pigmentos y pinturas en base de minerales de cobre inscribe su producción en la discusión sobre los procesos de explotación minera ocurridos desde tiempos prehispánicos en el norte de Chile. Los resultados muestran el uso de una diversidad de minerales, provenientes posiblemente de fuentes de materias primas diferentes, pero con tecnologías compartidas, en este caso entre las regiones de Antofagasta y Tarapacá. Más allá del mineral explotado, es indudable, que en tiempos prehispánicos la selección de los minerales de cobre para la producción de pigmentos y pinturas dependió del color deseado y del uso posterior en distintos soportes.

La incorporación a la reflexión de las producciones lapidarias y metalúrgicas nos parece fundamental para entender el uso de pigmentos y pinturas presentes en el desierto de Atacama y regiones vecinas. Los resultados obtenidos en el marco de otros proyectos en desarrollo sobre las pinturas rupestres del norte de Chile nos permiten pensar que esta producción fue una práctica de

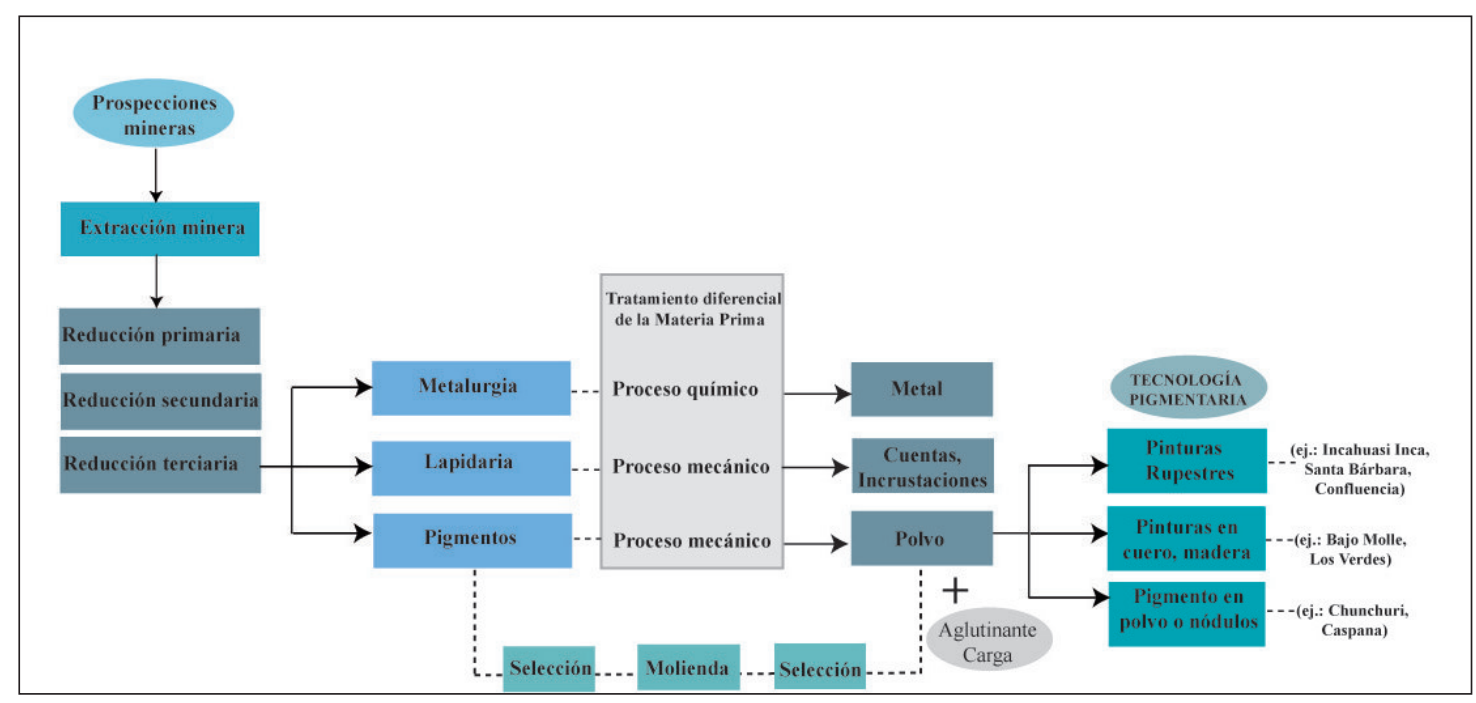

Figura 8. Cadena operativa de los pigmentos a base de minerales de cobre a partir del modelo esquemático de la cadena operativa minera definido por Salazar (2003-2004), y Salinas y Salazar (2008) (adaptado de Sepúlveda et al. 2013a). 
raigambre más bien local. Esto se ve reafirmado por el hecho que el uso de minerales de cobre se inserta dentro de una tradición minera más amplia y de larga data en las regiones de Antofagasta y Tarapacá. Más aún su producción habría permanecido en tiempos incaicos y coloniales, al menos hasta el siglo XVIII, contribuyendo a articular importantes redes de interacción en toda el área Centro-Sur andina.

Pese a eso, todavía faltan muchos elementos para poder reconstruir el panorama de la fabricación, la organización espacial de la producción, la distribución y el uso de los pigmentos fabricados a partir de minerales de cobre. Sin embargo, insistimos sobre el hecho de que esta tecnología pigmentaria debe considerarse como otro aspecto inherente a considerar en las cadenas operativas mineras, y que además debe ser tratado con la misma importancia que los otros componentes (lapidaria y metalurgia)
(Figura 8). Más aún, planteamos que esta reflexión debe continuar de modo de poder precisar aún más las formas de inserción de la tecnología pigmentaria en las formas de interacción que ocurrieron en el pasado en el desierto de Atacama.

Agradecimientos Investigación financiada por los proyectos FONDECYT de Iniciación 11130651 y ECOS-CONICYT C11Ho2. El Proyecto UTA 2014, 3711-14 contribuyó en la materialización de los análisis aquí presentados. Este trabajo no hubiese sido posible sin la enorme colaboración y amabilidad del Museo Regional de Iquique, en particular agradecemos a Francisco Téllez y a Patricio Muñoz por facilitarnos el acceso a las colecciones de dicha institución. Agradecemos igualmente a Lautaro Núñez por su ayuda en relación con la atribución de los contextos de la colección Anker Nielsen. Se reconoce finalmente el apoyo del Convenio de Desempeño UTA-MINEDUC.

\section{* Referencias citadas}

ADÁN, L. y M. URIBE. 1995. Cambios en el uso del espacio de los períodos agroalfareros: un ejemplo de ecozona de quebradas altas, la localidad de Caspana (Provincia de El Loa, II Región). Actas II Congreso de Antropología Chilena, vol. II: 541-555. Valdivia, Chile.

AJATA, R. y P. MÉNDEZ-QUIRÓS. 2012. Buscando el Formativo en la costa Tarapaqueña: prospección arqueológica y gestión de datos en sistemas de información geográfica. Actas del XVIII Congreso Nacional de Arqueología Chilena: 43-52. Valparaíso, Chile.

ARRIAZA, B. T., L. CORNEJO, H. LIENQUEO, V. G. STANDEN, C. M. SANTORO y J. ACARAPI. 2006. Caracterización elemental de arcillas utilizadas en la momificación artificial de la Cultura Chinchorro, extremo norte de Chile, mediante espectrometría de fluorescencia de rayos-X de energía dispersiva (EDXRF). Anales del X. Seminario Latinoamericano de análisis por Técnicas de Rayos $X$, editado por L. Cornejo, pp. 1-7. Universidad de Tarapacá-CIHDE, Arica.

ARRIAZA, B. T., L. CORNEJO, H. LIENQUEO, V.G. STANDEN, C.M. SANTORO y J. ACARAPI. 2008. Caracterización química de las arcillas utilizadas para la momificación artificial en la Cultura Chinchorro, extremo Norte de Chile. En Mummies and Science. World Mummies Research, editado por P. Atoche, C. Rodríguez y M. A. Ramírez, pp. 515-520. Santa Cruz de Tenerife.
AYALA, P., O. REYES y M. URIBE. 1999. El cementerio de los Abuelos de Caspana: el espacio mortuorio local durante el dominio del Tawatinsuyu. Estudios Atacameños 18: 35-54.

BERENGUER, J. 2007. El camino inka del Alto Loa y la creación del espacio provincial en Atacama. En Producción y Circulación Prehispánicas de Bienes en el sur Andino, editado por A. E. Nielsen, M. C. Rivolta, V. Seldes, M. M. Vásquez y P. H. Mercolli, pp. 413-443. Editorial Brujas, Córdoba.

BERENGUER, J. y I. CÁCERES. 2008. Los Inkas en el altiplano sur de Tarapacá: El Tojo revisitado. Chungara 40 (2): 121-143.

BERENGUER, J., C. SANHUEZAy I. CÁCERES. 2011. Diagonales incaicas, interacción interregional y dominación en el altiplano de Tarapacá, norte de Chile. En Ruta, Arqueología, Historia y Etnografía del Tráfico sur Andino, editado por L. Núñez y A. Nielsen, pp. 247-283. Encuentro Grupo Editor, Córdoba.

BIRD, J. 1977-78. El Hombre de Cobre, un minero prehistórico del Norte de Chile y sus herramientas. Boletín del Museo Arqueológico de La Serena 16: 77-106.

1979. The "Copper man": a prehistoric miner and his tools from northern Chile. En Precolumbian metallurgy of South America, E. Benson (Ed.), pp. 105-32. Dumbarton Oaks Research Library and Collection, Washington. 
CARRIÓN, H. 2010. Caracterización tecno-morfológica de cuentas de mineral de cobre durante el Periodo Medio en San Pedro de Atacama. Informe Final de Práctica Profesional. Departamento de Antropología, Universidad de Chile, Santiago.

CHAVEZ, W. X. JR. 2000. Supergene oxidation of copper deposits: zoning and distribution of copper oxide minerals. Newsletter Society of Economic Geologists 41: 9-21.

CERVELLINO, M. y N. SILLS. 2001. El arte rupestre de los sitios Finca de Chañaral y Quebrada de Las Pinturas, Región de Atacama. En Segundas Jornadas de Arte y Arqueología, pp. 134-151, J. Berenguer, L. E. Cornejo, F. Gallardo y C. Sinclaire (Eds.). Museo Chileno de Arte Precolombino, Santiago.

CIFUENTES, A. 2014. Metales y metalurgia en San Pedro de Atacama durante el periodo Medio: hacia la definición de una metalurgia local. Tesis para optar al Grado de Arqueólogo, Departamento de Antropología, Universidad de Chile, Santiago.

COQUINOT, Y., H. SALOMON. 2013. First Investigations into the mine San Ramón 15 and use of iron oxide rich materials, Taltal. Informe de Cooperación Internacional, proyecto FONDECYT 1110196, Año 2. (manuscrito inédito).

COQUINOT, Y., H. SALOMON. 2014. La mine de San Ramón 15, Taltal. Informe de Cooperación Internacional, proyecto FONDECYT 1110196, Año 3. (manuscrito inédito).

CRADDOCK, B.R. 1990. The experimental hafting of stone mining hammers. En Early mining in the British Isles, P. Crew and S. Crew (Eds.). Snowdonia National Park Study Center, London.

DOWNS, R. 2006. The RRUFF Project: an integrated study of the chemistry, crystallography, Raman and infrared spectroscopy of minerals. Program and Abstracts of the 19th General Meeting of the International Mineralogical Association in Kobe, Japan. Oo3-13.

FIGUEROA, V., I. MONTERO y S. ROVIRA. 2oloa. Estudio Tecnológico de objetos procedentes de Cerro Turquesa (San José del Abra, II región). Actas del XVII Congreso Nacional de Arqueología Chilena, pp. 1135-1147. Ediciones Kultrún, Sociedad Chilena de Arqueología, Universidad Austral, Valdivia, Chile.

FIGUEROA, V., D. SALAZAR, H. SALINAS, P. NÚÑEZ- REGUEIRO y G. MANRÍQUEZ. 2013. Prehispanic Mining Ergology of Northern Chile: An Archaeological Perspective. Chungara 45 (1): 61-81.

GAllardo, F., G. CABELlO, G. PIMENTEL, M. SEPÚlVEdA y L. CORNEJO. 2012. Flujos de información visual, interacción social y pinturas rupestres en el desierto de Atacama (norte de Chile). Estudios Atacameños 43:35-52.
GARCÍA-ALBARIDO, F. 2007. Umiñas en el Período Intermedio Tardío y el Tawantinsuyu: una aproximación a la lapidaria atacameña. Informe Final de Práctica profesional. Departamento de Antropología, Universidad de Chile.

GISBERT, T. 1994. El señorío de los Carangas y los chullpares del río Lauca. Revista Andina 12(2): 427-481.

GONZALEZ, C. 2007. Qhapacñan en el extremo meridional del despoblado de Atacama. Actas del XVI Congreso Nacional de Arqueología Argentina, Tomo II, pp. 511-518. Mendoza, Argentina.

GONZALEZ, C. y C. WESTFALL. 2008. Atacameños en El Salvador: nuevas apreciaciones sobre un fardo funerario del Cementerio Las Turquesas. Estudios Atacameños 35: 49-73.

GUZMAN, F., M. PEREIRA, M. SEPÚLVEDA, J. CARCAMO y S. GUTIERREZ. 2014. Las pinturas murales de la iglesia de San Andrés de Pachama, norte de Chile. Trabajo presentado en el Seminario International Materiality between art, science and cultu$r e$ in the Viceroyalties (XVI-ХVIII centuries): an interdisciplinary visión towards the writing of a new colonial art history, Buenos Aires-Córdoba. Marzo 2014. (manuscrito en poder de los autores).

HELWIG, K. e I. WAINWRIGHT. 1999. Análisis of pigment simples, Río Loa subregion, Loa Superior Region, Northern Chile. Informe Final Proyecto FONDECYT 1980200. (manuscrito inédito).

LYNCH, T.F. y L. NÚÑEZ. 1994. Nuevas evidencias inkas entre Kollahuasi y Río Frío (I y II Regiones del Norte de Chile). Estudios Atacameños 11:145-164.

Mille, B., D. SAlAZAR, C. PERLES, J. BERENGUER y V. FIGUEROA. 2013. Rapport année 2 Projet ECOS-CONICYT $\mathrm{C}_{11} \mathrm{H}_{12}$ "La préhistoire du cuivre: une approche comparative de l'organisation de la production métallurgique. (manuscrito en poder de los autores).

NEUMANN, H. 1973. Mineralizaciones tipo cobre porfídico en Chile. Revista Geológica de Chile 1: 67-73.

NÚÑEZ, L. 1966. Recientes fechados radiocarbónicos de la arqueología del norte de Chile. Boletín de la Universidad de Chile 64-65:32-41.

1987. Tráfico de metales en el área centro-sur andina: factos y expectativas. Cuadernos del Instituto Nacional de Antropología 12: 73-105.

1999. Valoración minero-metalúrgica circumpuneña: menas y mineros para el Inka Rey. Estudios Atacameños 18: 177-221.

2005. La naturaleza de la expansión aldeana durante el Formativo tardío en la cuenca de Atacama. Chungara 37(2): 165-193. 
NÚÑEZ, L. y T. DILLEHAY. 1995. Movilidad Giratoria, Armonía Social y Desarrollo en los Andes Meridionales: Patrones de Tráfico e Interacción Económica (Ensayo). Universidad del Norte, Antofagasta.

REES, C. 1999. Elaboración, distribución y consumo de cuentas de malaquita y crisocola durante el período Formativo en la vega de Turi y sus inmediaciones, subregión del río Salado, norte de Chile. En Los tres reinos: prácticas de recolección en el cono sur de América, C. Aschero, M. A. Korstanje et P. M. Vuoto (Eds.), pp. 83-93. Magma publicaciones, Buenos Aires.

SALAZAR, D. 2002. El complejo minero San José del Abra, II Región (1450-1536 DC). Una aproximación a la Arqueología de la Minería. Tesis para optar al grado de Magíster, Facultad de Ciencias Sociales, Universidad de Chile. Santiago de Chile.

2003-2004. Arqueología de la minería: propuesta de un marco teórico. Revista Chilena de Antropología 17: 125-150.

SALAZAR, D. y H. SALINAS. 2008. Tradición y transformaciones en la organización de los sistemas de producción mineros en el norte de Chile prehispánico: San José del Abra, siglos I al XVI d.C. En Minas y metalurgia en los Andes Centrales y del Sur. Desde tiempos prehispánicos hasta el siglo XVII, P. Cruz, P. Absi y M. Van Buren (Eds.), pp. 163-200. IRD/IFEA/University of Colorado State/ ABNB, Sucre.

SALAZAR, D., H. SALINAS, V. MCROSTIE, R. LABARCA y G. VEGA. 2010. Cerro Turquesa: diez siglos de producción minera en el extremo norte de Chile. Actas del XVII Congreso Nacional de Arqueología Chilena, Tomo II, pp. 1085-1097. Ediciones Kultrún, Sociedad Chilena de Arqueología, Universidad Austral, Valdivia, Chile.

SALAZAR, D., D. JACKSON, J.-L. GUENDON, H. SALINAS, D. MORATA, V. FIGUEROA, G. MANRÍQUEZ y V. CASTRO. 2011a. Early Evidence (ca. 12,000 BP) for Iron Oxide Mining on the Pacific Coast of South America. Current Anthropology 52(3): 463-475.

SALAZAR D., V. FIGUEROA, D. MORATA, B. MILLE, G. MANRIQUEZ y A. CIFUENTES. 2olıb. Metalurgia en San Pedro de Atacama durante el Período Medio: Nuevos datos, Nuevas Preguntas. Revista Chilena de Antropología 13 (1): 123-148.

SALAZAR, D., J. BERENGUER y G. VEGA. 2013. Paisajes minerometalúrgicos inkaicos en Atacama y el altiplano sur de Tarapacá (norte de Chile). Chungara 45 (1): 83-103.

SALINAS, H. y D. SALAZAR. 2008. Cadenas operativas y sistemas de explotación minera prehispánica. En Puentes hacia el pasado: reflexiones teóricas en arqueología, D. Jackson, D. Salazar y A. Troncoso (Eds.), pp. 73-91. Serie Monográfica de la Sociedad Chilena de Arqueología 1, Santiago.
SANHUEZA, J. 1985. Poblaciones tardías en playa Los Verdes, costa sur de Iquique, I Región, Chile. Chungara 14: 45-60.

SEPÚLVEDA, M. 2006. Art rupestre et iconographie dans le bassin de la rivière Salado (nord du Chili), à la période Intermédiaire Tardive (850-1450 apr. J.-C.). Tesis para la obtención del Grado de Doctor en Prehistoire, Ethnologie et Anthropologie, Université Paris I Panthéon-Sorbonne, Francia.

SEPÚlVEDA, M., V. FIGUEROA y S. PAGÉS-CAMAGNA. 2013a. Copper pigment- making in the Atacama Desert (northern Chile). Latin American Antiquity 24(4): 467-482.

SEPÚlVEDA, M., S. GUTIÉRREZ, M. CAMPOS-VALLETTE, E. CLAVIJO, P. WALTER y J. J. CÁRCAMO. 2013b. Raman spectroscopy and X-ray fluorescence in molecular analysis of yellow blocks from the archeological site Playa Miller 7 (northern Chile). Journal of the Chilean Chemical Society 58(3): 1651-1654.

SEPÚLVEDA, M. y E. LAVAL. 2o10a. Uso de minerales de cobre en la pintura rupestre de la localidad del río Salado (II región, norte de Chile). Actas del XVII Congreso Nacional de Arqueología Chilena 2006. Tomo II, pp. 1111-1124. Ediciones Kultrún, Sociedad Chilena de Arqueología, Universidad Austral, Valdivia, Chile.

SEPÚLVEDA, M. y E. LAVAL. 2o1ob. Aplicación y aplicabilidad de métodos físico- químicos para el estudio de las pinturas rupestres. Ejemplo de estudio en la localidad del río Salado (II Región, norte de Chile). Actas del XVII Congreso Nacional de Arqueología Chilena 2006, Tomo II, pp. 825-834, Valdivia.

SEPÚlVEDA, M., E. LAVAL, L. CORNEJO y J. ACARAPI. 2012. Elemental characterization of prehispanic rock art and arsenic in northern Chile. Rock Art Research 29 (1): 93-107.

SEPÚlVEDA, M., H. ROUSSELIERE, E. VAN ELSLANDE, B. ARRIAZA, V. STANDEN, C. SANTORO y P. WALTER. 2014. Study of color pigments associated to Archaic mummies and grave goods in Northern Chile (7.000-3.500 B.P.). Journal of Heritage Science 2: 7 .

SILVA-PINTO, V., D. VALENZUELA, M. SEPÚlVEDA. 2013. Osteaortrosis poliarticular en Chinchorro. Revisión de un caso y discusión sobre el autocuidado en la prehistoria de Arica, norte de Chile. Revista Médica de Chile 141 (5): 644-650.

SIRACUSANO, G. 2005. El poder de los colores. De lo material a lo simbólico en las prácticas culturales andinas. Siglos XVI y XVII. FCE, Argentina. 
SOTO, C. 2010. Tipología de cuentas de collar en la quebrada de Tulán (Salar de Atacama): nueva línea de evidencia para la transición arcaico-formativo. Actas del XVII Congreso Nacional de Arqueología Chilena 2006, Tomo II, pp. 1123-1134. Ediciones Kultrún, Sociedad Chilena de Arqueología, Universidad Austral, Valdivia, Chile.

TOMASINI, E., C. RÚA, G. SIRACUSANO y M. MAIER. 2012. Atacamite as a natural pigment in a South American colonial polychrome sculpture from the late XVI Century. Journal of Raman Spectroscopy 44(4): 637-642.

UHLE, M. 1912-1913. Informe presentado sobre el viaje de exploración arqueológica hecha en la expedición a Calama. Anales de la Universidad de Chile. Boletín 132 (marzo-abril): 95-10o.

URBINA, S. 2012. "Asentamientos y autoridades en Tarapacá durante los siglos prehispánicos tardíos y coloniales tempranos (s. X-XVII d.C.)”. Tesis para optar al grado de Magíster en Etnohistoria, Facultad de Filosofía y Humanidades, Universidad de Chile.
URBINA, S., L. ADÁN, C. MORAGAS, S. OLMOS y R. AJATA. 2011. Arquitectura de asentamientos de la costa de Tarapacá norte de Chile. Estudios Atacameños 41: 63-96.

URIBE, M. 2009. El período Formativo de Tarapacá y su cerámica: avances sobre complejidad social en la cosa del Norte Grande de Chile (900 a.C.-80o d.C.). Estudios Atacameños 37: 5-27.

WESTFALL, C. y C. GONZÁLEZ. 2010. “Mina Las Turquesas: un asentamiento minero lapidario preincaico en el extremo meridional circumpuneño, Región de Atacama, Chile". Actas del XVII Congreso Nacional de Arqueología Chilena, Tomo II, pp. 1073-1082. Ediciones Kultrún, Valdivia.

ZORI, C. 2011. "Metals for the Inka: Craft Production and Empire in the Quebrada de Tarapacá, Northern Chile". Tesis Doctoral. University of California Los Angeles, California.

ZORI, C. y P. TROPPER. 2010. Late pre-hispanic and early colonial silver production in the Quebrada de Tarapacá, Northern Chile. Boletín del Museo Chileno de Arte Precolombino 15: 65-87. 